\title{
On the semantic feature structure of modeling concepts: an empirical study
}

\author{
Dirk van der Linden*† ${ }^{* \dagger}$ Marc van Zee ${ }^{\ddagger}, \S$ \\ * Public Research Centre Henri Tudor, Luxembourg \\ $\dagger$ Radboud University Nijmegen, the Netherlands \\ $\ddagger$ University of Luxembourg, Luxembourg \\ $\S$ EE-Team, the Netherlands
}

Contact:dirk@ee-team.eu,marc.vanzee@uni.lu

\begin{abstract}
Is an actor typically considered a human being? What about an autonomous entity? We investigate the typical feature structure of common modeling concepts in order to create an empirically grounded description of the semantic feature structure that people implicitly use while reasoning about, and with such concepts. Apart from the insights into modeling concept structure that this work presents, consequences for the quality of models and use of modeling languages are discussed. We finally discuss in more detail how the process of modeling, especially when it involves multiple people with different backgrounds, modeling different aspects (i.e., enterprise modeling), stands to benefit from more insights into how the individual modelers see the basic modeling concepts shared between them.
\end{abstract}

\section{INTRODUCTION}

Some basic concepts (e.g., actors, goals, resources) used in conceptual modeling can be found in most modeling languages. However, while these concepts might superficially appear to be the same, they are often interpreted in subtly different ways by different modelers (because of their background, focus, or intention during modeling). As such, different modelers will likely hold, however slight, different understandings of the same concepts. By concepts we mean the understanding that someone has of the thing they are using to model, which might be different than the stipulated understanding (e.g., the semantics attributed to some concept by standards, a language specification, or some group consensus process). This is an issue for several reasons, especially in collaborative efforts like Enterprise Modeling, because models created by people with different understandings are often integrated [1], linked [2], or used to communicate between people. It is thus important to be aware of the interpersonal differences in understanding of concepts that might superficially seem similar, as otherwise integrated models or communication might be semantically inconsistent and not represent the originally intended semantics (cf. [3]). Understanding these interpersonal differences is a multi-faceted issue, as there are several aspects of personal conceptualizations that have to be studied and understood. For example, the structure of a concept in modelers' minds can be discrete or continuous, leading to different kinds of comparison judgments, the elements that are most important to someone differ from person to person, and the features used subconsciously to identify something as being 'that' concept or not differ between (groups of) people. We will focus here on the features that people use to identify things as being (a good example of) a concept or not. For example, whether "John
Doe working in the Sales department overseeing a particular business process" is an actor in the context of that business process or not.

It is important to note that in this paper we take a subjectivist stance, in the sense that we assume all involved modeler's and stakeholder's conceptualizations are inherently interesting and potentially worthwhile to the modeling effort, more so than assuming there is a single golden standard of semantics all ought to adhere to. This holds especially for efforts like enterprise modeling, where there are so many stakeholders and modelers involved. Because of this the actual practice of modeling becomes an inter-subjective activity [4], [5], [6], where the act of modeling comes down to finding a good representation of the shared social reality.

The rest of this paper is structured as follows. We elaborate on the theoretical background of concepts in Section II, clarify the relevance for (conceptual) modeling in Section III, and state our research objectives in Section IV. In Section V we will elaborate on the method and data used in our empirical study. We briefly summarize the results in Section VI, and discuss them in more detail in Section VII. Finally we conclude in Section VIII and include complete datasets for others to use in the appendices A through A.

\section{BACKGROUND}

Things are identified by their features, whether they are physical objects, abstract elements or psychological entities like concepts [7], [8]. For example, I might identify a certain object as a table because it has some properties I have come to expect of tables (e.g., it has four legs, is made of wood, is of the right height to sit at, and there happens to be a full set of dinnerware on top of it). This holds for conceptual entities as well, where, for example, we identify the creation of a good research paper as a goal because we see certain features like wanting to achieve it, the possibility of it being achievable, and we want to achieve it, that it can be achieved, and that there is some way to achieve it. The features we identify things by are often of a structural (an aspect of a thing's physical or logical composition: the table being made of wood) or functional (an aspect of what a thing is used for, or does: the table being used to eat at) nature. Earlier work [9] found that non-natural concepts (i.e., human-made or abstract things) were also mostly identified by their structural features, 
as examples with similar functionality but different structure were still excluded as being part of those concepts. This might mean that the modeling concepts we use are as well strongly identified by their structural features (e.g., a process being composed of steps, an actor being a human thing, a resource being physical material).

This kind of identification through features is fundamental to human nature [10], as we categorize everything around us. It is how we decide just what things are. When something does not have the properties we typically associate with it, or differs from them too much, we simply reject it as possibly being so. For example, a typical Japanese dining table would be far less of a typical dining table for most Europeans given the difference in structure (being significantly lower), and function (being interacted with in a fundamentally different way: sitting on the floor in front of it instead of on a chair). As such, two different people could have a fundamental disagreement about whether a specific thing is a table or not. The identification of things in this way is an important aspect of determining whether something corresponds to our expectations - whether it is a correct model of our subjective reality. Such judgments are not discrete, but most often graded: things are not simply considered to 'be' a thing or not, but they are so to a certain degree. When we see a particular thing with some set of features (for instance the Japanese table) we thus make a graded judgment deciding to what degree it is a table or not. Whether this is done in the brain by comparing to an abstract set of features (in case of prototype theory), or to specific exemplars with a set of features is (in case of exemplar theory) is still undecided. However, it is clear that a comparison to some set of observed features is made.

The basic modeling concepts we use so often in practice are no different from this. For example, someone could simply find it incorrect to view a 'department' as being an actor, because they are not singular entities human entities that can easily be attributed moral responsibility for their actions. When that person is forced to model a department as an actor in some modeling language, because the language dictates it to be done in such a fashion, the produced model is not a truly valid representation of that modeler (or stakeholder's) conceptualization anymore. If we had more of an insight into what features are most common and typical for these modeling concepts we would have a better chance of judging whether a produced model corresponds to the involved modelers' and stakeholders' conceptualizations. Perhaps more importantly, if we know how stakeholders and modelers alike identify things in terms of their features (i.e., what the modeling concepts really are for them), we could reason whether the modeling languages we use let us (or can be extended to) model the world as they see it.

\section{RELEVANCE FOR (CONCEPTUAL) MODELING}

Investigating what features are most linked with common modeling concepts is thus directly relevant to the practice of conceptual modeling. In particular, the quality of models and modeling languages can benefit from a deeper understanding into these features, as it would be easier to ensure models are a valid representation of the conceptualization someone has of a particular domain. While quality is a term with many different meanings and aspects [11], [12], determining whether a model is able to convey the intended meaning of the original modeler [13] is surely relevant for all purposes. This holds especially when models are used for building and exchanging knowledge [14] (i.e. communicating between different parties), as is often the case with the different aspects modeled in Enterprise Modeling, making it important to be aware of the validity of just what knowledge is being exchanged.

An empirically derived understanding of the features that modelers typically associate with modeling concepts would help us in this, especially so because we tend to model with the semantics of our own natural language [15], and all the bias that comes with doing so. The primary use would be in knowing how people (in the context of conceptual modeling) actually judge whether some element from the universe of discourse is a typical example of some concept, or whether it is barely so. For example, if resources are typically resources because they are material, we know that we need to be very careful when we want to model an abstract piece of 'information' as a resource for some business process, and perhaps discuss whether we select instead a material representation of that information (i.e., a set of documents). By analyzing what kind of features are used to make these judgments (e.g., structural or functional, or other yet unknown modalities), we could also gain more insight into the fundamental nature of these concepts themselves. Furthermore, from analyzing the most typical features (e.g., those often repeated or shared between different modelers) and atypical features, we could find specific areas that would benefit from extra attention during modeling sessions. If we know what there are a large number of varied views for a particular concept (e.g., many people holding different views on what constitutes a resource), focusing extra discussions during the modeling process on those concepts would help enhance the validity of produced models, and likely have a positive effect on the agreement between the people involved [16]. Finally, knowing what features are most typical would make it possible to analyze where modeling languages might go wrong (by forcing people into an atypical use of a concept), or can be made better (by not allowing for the explicit modeling of some particular conceptualization). Our modeling languages should be discriminatory enough in their notation to provide clues when certain concepts truly mean different things, i.e., when the concepts they describe have (significantly) different feature-sets.

\section{RESEARCH OBJECTIVES}

However, before we can begin to think and act on implementing such changes and improvements, we need data on modelers and their conceptual idiosyncrasies. We should investigate on a more fundamental level how these people view modeling concepts in terms of necessary and sufficient features, what is most typical, and what is absolutely not. Only then can we carefully attempt to improve our models and modeling languages, ensuring they are truly valid representations of the conceptualizations held by modelers and stakeholders. As Goddard [17] wrote about research into word meanings and concepts:

"Given that word meanings are psychologically real concepts that people use to categorize, organize, and 
communicate about their mental lives, they ought to be a valued object of study in their own right." [17]

The field of conceptual modeling has typically been involved with the representation of concepts and conceptual information [18], so taking such a focus seems justified. We will focus specifically on performing a thorough investigation into the feature sets which conceptual modelers use for the identification of basic modeling concepts. Conceptual modeling as a field of research often lacks fundamental work which observes and tests [5], which is sorely needed [19], instead focusing more on designing solutions and artifacts. Our goal is to provide an empirically grounded detailed list of features and analysis which practitioners and researchers can use for further research and modeling efforts, rather than 'jumping' to the suggestion of how any and all modeling should be done. Concretely, our research questions are:

1) Which features do conceptual modelers use for the identification of common modeling concepts?

a) Are such features of structural or functional nature?

b) What other modalities can be found in such features?

2) Are there typical features shared between multiple conceptual modelers?

\section{METHOD}

In this section we give an overview of the exact procedure we followed for our study, what materials we used, how we gathered the participants, and how we processed the results. We chose to perform a simple elicitation study with clear semantic priming at every question, where it was made clear to participants that all questions and answers were to be given in the context of conceptual modeling.

\section{A. Materials}

The basic examples of a chair and its functional and structural features were inspired by common works in cognitive science and categorization studies (e.g., [20]). The concepts we selected (ACTOR, EVENT, GOAL, PROCESS, RESOURCE, RESTRICTION, RESULT) derived from previous work into the analysis of modeling languages used for (different aspects modeled in) enterprise modeling (see [21]). We used descriptions of the concepts that were wide enough to not prime participants on a particular interpretation.

\section{B. Participants}

We invited professionals from academia and practice with significant conceptual modeling experience to participate in our study. Where available, we invited people who had participated in our earlier studies, and had at least an intermediate level of experience with modeling and modeling languages. As we did not contact each potential participant individually, but did so by requesting intermediaries (e.g., managers, team leaders) to set out the survey among their employees, we cannot give the total amount of people contacted. In total 45 people participated, predominantly from industry, all of which were experienced modelers employed at various internationally operating businesses located throughout Europe and the United
States. All participated voluntarily and received no compensation for their participation.

\section{Procedure}

The survey was executed online. Participants were instructed in detail what the experiment was about, and what kind of information was needed. First we elicited what modeling languages the participant had significant or extensive experience with, followed by a longer explanation of the feature questions. For the studied set of seven modeling concepts we then asked participants to write down any and all features they would typically attribute to this concept, in the context of modeling. In order to encourage as much intuitive responses, participants were allowed to respond such features in a number of major languages locally used (English, German, French, Dutch). The results of this step were first (where necessary) translated into English, and then iteratively analyzed in order to standardize the grammatical and lexical form of the feature (e.g., standardizing 'human', 'is human', 'a human thing' to 'is human'). After this, another iteration followed in which features with the same meaning were clustered together (e.g., 'is a person', 'is a human being', 'is a human' into 'is human'). We then counted the amount of times that a feature was expressed by participants in order to determine which features were most commonly given. Each of these features were also analyzed to determine their modality and whether they were of a structural or functional nature (e.g., a RESTRICTION 'limiting behavior' is functional, while 'exists as an abstract thought' is structural.).

\section{RESULTS}

The diverse amount of views on the different concepts we investigated was ensured by the wide variety of participants and the languages they were specialized in. While most common were general purpose language such as UML, ER and their variations, ArchiMate was also widely used. The total list of languages participants claimed to use (see Table I) encompasses a wide range including also a host of formalized languages, methods and frameworks, and improvisational approaches (e.g., sketching models with pen and paper or in Microsoft PowerPoint).

TABLE I. USED MODELING LANGUAGES.

\begin{tabular}{l}
\hline language \\
\hline Object-oriented programming languages, IDEF, UML, ER, ArchiMate, BPMN, \\
NIAM, Essence, Petrinets, BPEL, Amber, Merode, Turtle, DEMO, Dataflow dia- \\
grams, The Decision model visual modeling for decision logic, EPC, IE, CBM, BMC, \\
TOGAF, IDEF, IDEF0, IT City Planning, Flowcharts, Value stream mapping, Value \\
chain diagram, SIPOC, BPEL, ARIS, Proprietary language, Microsoft Powerpoint, \\
ORM, Business object diagrams, Natural language, Plateu planning, ISAC, Yourdon \\
Ward en Mellor ERD, Evolutionary NIAM, CaseTalk, JBF, i*, Secure Tropos, \\
CORAS, VCL, VHDL, WSDL, e3Value, RBAC, XACML, EMF, BMO, PERT. \\
\hline
\end{tabular}

As the complete, raw list of elicited features is too large to treat here (see Table III for their respective sizes), we give an overview of cleaned up results, with redundant features removed from them in the appendices. We will focus specifically on the reoccurring features (i.e., those likely more typical as they were shared between multiple participants), which are given in Table II. An overview of the different modalities expressed in the results is given in IV. Full raw datasets, including pre-translated and normalized data are available upon request. 


\section{DISCUSSION}

In this section we will first come back to the research questions we stated, and then discuss some of the more interesting findings in more detail.

Which features do conceptual modelers use for the identification of common modeling concepts? and Are there typical features shared between multiple conceptual modelers? The results given in appendices A through A are a complete, processed overview of all the elicited features that conceptual modelers use to identify common concepts. They are unweighted terms which, for at least one practitioner are in the top 5 most important features which they use to identify that specific concept by. For example, in appendix A the first features are 'has a name', 'has permissions', 'has a role', and so on. This means that, in order to be a conceptually valid actor, an entity should have some kind of name that identifies it, it needs to have permissions on something, and it needs to hold a specific role. The total amount of terms here is a collection of the results from all the individual participants and is thus not meant to be a requirement for what every actor has to be (the individual feature sets for each participant could not be included because of space constraints). A more targeted understanding of that can be found in Table II, where only the features that were repeated by multiple participants are listed. Almost all of the concepts have at least some repetitions of features that allow for identification, whether it is the existence of some specific name or label, or them being capable of being identified or observed. Furthermore, most concepts can be dealt with on an instantiation level, as often they are seen in terms of specific instantiations.

TABLE II. THE MOST OFTEN REOCCURRING FEATURES WE ELICITED IN OUR STUDY. THE COMPLETE DATASET CANNOT BE INCLUDED DUE TO SPACE CONSTRAINTS, BUT IS AVAILABLE UPON REQUEST.

\begin{tabular}{ll}
\hline concept & feature \\
\hline ACTOR & has a name, has a role, has skills, can perform an action / actions, \\
& has a responsibility / responsibilities, has capabilities, carries \\
& knowledge, has permissions, has rights, is human, is independent, \\
& carries out actions, is machine, is a person (with a role in a \\
& company), is an organization, initiates actions, is autonomous, is \\
& active, has responsibilities, is part of a business function \\
EVENT & is triggered by something, has consequences, has a trigger, can \\
& trigger an action, has a duration, triggers a process, occurs at a \\
& time, can be observed, has a name, happens, is a process end, \\
& triggers a task, has a description, has a source, is atomic, is a \\
& trigger, is repeatable, is an occurrence, is temporal, can trigger a \\
process & has a related stakeholder, has a name, can be achieved, becomes \\
an evaluation criteria for future accomplishments, is clear, is \\
measurable, has to be achieved, gives direction, is desired, is \\
PROCESS & in the future \\
RESOURCE & is used for something, is material, is immaterial, is countable, is \\
& scarce, has a name, has a value, has costs, has a source, can be \\
& human, is human, is a machine, is needed for an activity, has a \\
type & has a type, limits something, has a name, has conditions, is \\
natural, limits something, limits possible solutions, is quantitative, \\
RESTRICTION \\
has a source, is measurable, can be temporal \\
is measurable, has a name, has a quality, has a description, is \\
realized, is observable, has value for someone
\end{tabular}

The most conserved features for the concept actor center around, as could be expected, its ability to act. Specifically its logical and/or ethical capability to act when it should do so (e.g., having responsibilities), and its role of acting as an independent or autonomous entity. This seems to point the common view of an actor more towards human beings and (smart) agents, not simple reactive agents or automata. Furthermore, this is expressed again in the often reoccurring features that identify exactly what acts, including such features as stating whether it is a human being, a specific person, a machine or an organization. When it comes to events, the most conserved features are that of being a trigger for something, and needing to be triggered itself. This shows that most modelers conceptualize of events as intentional things: things that are specifically made to happen. Environmental or contextual events, even if they might impact on something (e.g., some market conditions impacting a process' efficiency) are thus typically not considered to be events in this context, which is in line with the often used assumption of closed world semantics. Goals are most importantly identified by knowing whether they can be achieved, and to what degree they can be verified to have been achieved. This is in line with the often used distinction between hard and soft goals, as it is often important to know whether a goal's satisfaction criteria can actually be known to have been met. Processes are primarily identified as things with a clear, middle, and beginning, and having related actors that likely drive the events. For resources there are a number of important features. They are identified by their nature (e.g., material or immaterial), the availability (e.g., scarcity) and the need of them having to be used for something. This implies strongly a view that resources exist primarily as a contextualized entity that only exists in the context of some other entity (e.g., a process) using them for a specific purpose. Restrictions are, like most other features identified by their ability to be properly identified, described, and measured. However, this goes into more detail than some other concepts, as features like the specific conditions of what it limits and, and for how long it does so are found to be important for something to be a restriction. Results are quite similar to restrictions in terms of their identifying features: the ability to be properly identified, described, temporal conditions like when a result is achieved. Furthermore, just like resources it seems that results are another contextually existent entity, that they exist in the context of being the result of some specific thing, often a process. The feature sets described here are only a small set of the total elicited features. Of course all of the listed features, even those not repeated by others, are used by practitioners in actual situations for identification of concepts, and should thus be taken seriously as possible restrictions of what specific modeling concepts can be in professional contexts.

TABLE III. AMOUNT OF FEATURES ELICITED PER CONCEPT. AMOUNTS ARE GIVEN EXCLUDING REPEATED FEATURES, THE NUMBER OF WHICH ARE GIVEN AT THE BOTTOM OF THE TABLE.

\begin{tabular}{llllllll}
\hline & actor & event & goal & process & resource & restriction result \\
\hline total & 131 & 98 & 97 & 51 & 105 & 75 & 79 \\
$\%$ structural & $52 \%$ & $70 \%$ & $82 \%$ & $73 \%$ & $82 \%$ & $52 \%$ & $85 \%$ \\
$\%$ functional & $43 \%$ & $30 \%$ & $18 \%$ & $27 \%$ & $18 \%$ & $48 \%$ & $15 \%$ \\
\hline repetitions & 46 & 35 & 13 & 4 & 28 & 16 & 8 \\
\hline
\end{tabular}

Are such features of structural or functional nature? As Table III shows, the largest amount of features we elicited were of a structural nature, excepting the concept of actor, which also had a significant amount of functional features (likely because actors are inherently associated with what they do: their functionality). Most of the features that can be seen in the other Tables corroborate this quite clearly, as most often they deal with some structural aspect a concept has (e.g., having a label, being some specific kind). This is in line 
with the findings from Malt and Johnson where it was also found that most artifactual categories were identified primarily by structural features. The reason given for this is that most concepts have multiple structural features by which we identify them, but often only few main functions that we associate with them, thus giving rise to less functional features elicited from the individual participants. This means also that these feature sets are likely less (modeling task) context-dependent than if they were identified primarily by functional features, as the structure exists independent of the actions taken or the environment the entity is found in.

What other modalities can be found in such features? Apart from the distinction between structural and functional features, we found some reoccurring modalities expressed in the dataset. Table IV gives an overview of these, with some specific examples. A large group contains features of alethic nature, which talk about things that are or are not the case (both functional and structural). For example, an actor being a human thing, an event being triggered by something. These types of features clearly state that some feature has to be the case, and thus needs to be true for some thing in order for it to be that concept.

Some temporal features were also present in the data set, dealing with features having specific time-bound aspects. These temporal features are sometimes a stronger version of a similar alethic feature, like for example with the feature 'an actor always has an input', which is a stronger version of the logical requirement that 'an actor has an input'. Others are interesting because they transform structural features of some concepts, like a goal becoming a specific thing (e.g., an evaluation criteria) which specializes (and perhaps narrows) the set of needed and sufficient features for it to be a goal.

The other largest class of features we found were those of a modal nature. These deal with features that should be the case, like a 'resource has to be protected', 'an actor has to work under constraints'. The difference of these features compared to their alethic counterparts is that they are less strong in stating what is the case in the subjective reality of the modeler, and are thus likely weaker features for rejecting something as not being a proper instantiation of a concept. In this case, even though a resource has to be protected, some particular thing that is not protected, but has enough other features that correlate with resources, will likely still be identified as being a resource. Thus, the features of modal nature are less strong when it comes to rejecting the identification of something as a specific concept.

Finally, we found some mereological features, which express part-whole relationships. For example, the 'an actor is part of a business function' is stronger than a similar subset/type, as here the business function cannot exist without the actor. However, compared to the total amount of results these kind of features were few and far between and thus likely are not widespread amongst practitioners. It is interesting that the amount of mereological features is so low, as from some other features they could be inferred (for instance, if an actor is an absolutely necessary thing for some process, then it makes sense to see that actor as a part of the whole business process; the process collapsing and not existing without that actor).

\section{A. Further use of our findings}

In this section we aim to briefly touch upon what practical use the theoretical findings presented in this work could have.

Steering the communication in the modeling process. When we model, we communicate and interact with many people. Given this inter-subjective nature, many potential communication errors and terminological mismatches are bound to occur. Anything that helps us find potential weak spots where such events are likely to occur before committing models to paper would be a useful venture. Doing so can prevent far more costly rectifications in later stages, when models cannot simply be amended (e.g., when a business process has actually been implemented, when a software program has been made, when specific domain experts or stakeholders have moved on and are no longer available).

What we can take from the study presented in this paper is that individual modelers have typical conceptual understandings of the concepts they use to model elements from a domain with. The actual core of these conceptual understandings, i.e., the most conserved features, come to be so by a lifetime of experience, and are unlikely to just change during a single session in which people communicate and share their understandings. This correlates with findings from an earlier study [22] performed on how people deal with picking up a new process modeling language without being formally taught them. In this study it was shown that if someone was familiar with one particular language, the threshold to go to a different, similar one was very low (e.g., switching from one goal modeling notation to another). As many of such similar languages often have a strong overlap in their conceptual make-up, people who are strongly familiar with one can easily pick up the others because it matches their conceptual understanding, even if they have not mastered its specific syntax or visual notation yet. While the categorization judgments we make are often of a graded nature (for example something being an actor to a certain degree), meaning that there is a certain amount of flexibility between people holding different conceptual understandings, it should be clear that it is be worthwhile to investigate just what these typical understandings for people are as to preemptively avoid terminological or conceptual mismatches. As the most often found kind of feature in our datasets were those of an alethic or and structural nature, people seem to identify concepts very often by what they actually are for them. This ties in strongly to the use of ontologies during modeling work, especially when those ontologies are actual reflections of the understanding involved people have of the domain [23], for which studies like these can give valuable input. Such ontologies are often useful to define the specific fine-grained semantics modelers hold of the concepts they use for a particular task, which is especially useful given that most often languages like ER and UML are used [24], in which we need to stereotype and define these detailed semantics in order to properly document them.

As tempting as it would be to then start generalizing, and infer that particular groups or kinds of people will 'think' in a particular way about concepts, this usually does not hold. We found in an earlier study on the structure of practitioners' conceptual understandings [25] that groupings based on properties like background, used languages and techniques, aspects they operate in, and so on, were simply not correct. Nonetheless, 
TABLE IV. THE MAIN MODALITIES WE FOUND FEATURES OF IN THE ELICITED FEATURES. WE DISTINGUISH BETWEEN ALETHIC (WHAT IS OR HAS TO BE), TEMPORAL (WHAT WILL BE), DEONTIC (WHAT SHOULD BE), AND MEREOLOGICAL (WHAT IS PART OF WHAT).

\begin{tabular}{|c|c|c|}
\hline modality & feature prefix & example of feature \\
\hline \multirow[t]{3}{*}{ alethic } & is $\ldots$ & $\begin{array}{l}\text { an actor is human, an event is triggered by something, a goal is based on an objective, a process is composite, a resource } \\
\text { is used by an actor, a restriction is valid until some time, a result is material }\end{array}$ \\
\hline & has ... & $\begin{array}{l}\text { an actor has capabilities, an event has consequences, a goal has a related objective, a process has steps, a resource has } \\
\text { a quality level, a restriction has to be made explicit, a result has value for someone }\end{array}$ \\
\hline & does not ... & an event does not have a duration \\
\hline temporal & $\begin{array}{l}\text { always has ... } \\
\text { becomes ... }\end{array}$ & $\begin{array}{l}\text { an actor always has an input, an actor always has an output } \\
\text { a goal becomes an evaluation criteria for future accomplishments }\end{array}$ \\
\hline \multirow[t]{3}{*}{ deontic } & has to $\ldots$ & $\begin{array}{l}\text { a resource has to be protected, a goal has to be fulfilled, an event has to be monitored, an actor has to work under } \\
\text { constraints }\end{array}$ \\
\hline & can be ... & $\begin{array}{l}\text { an actor can be human, an event can be caught by something, a goal can be refined into a subgoal, a resource can be } \\
\text { available }\end{array}$ \\
\hline & may ... & $\begin{array}{l}\text { an event may be notified to listeners, a goal may be quantifiable, a process may involve roles, a resource may be } \\
\text { decomposed }\end{array}$ \\
\hline mereological & is part of ... & an actor is part of a business functional \\
\hline
\end{tabular}

in our experience during workshops with practitioners and companies operating the modeling domain we found that they still do often group modelers together on such assumptions, without necessarily ensuring they actually think the same way. We hope that the study we report on here provides a starting point for other researchers and modelers to deal with the need for explicit discussions of how we understand even basic things in detail.

Checking whether modeling languages accommodate combinations of features. The importance of having these features be explicitly accommodated by a modeling language is because they represent potential conceptual misunderstandings which might otherwise go unnoticed. In earlier work [26] we explored roughly what concepts and distinctions are accommodated implicitly or explicitly in a number of often used domainspecific modeling languages. However, how should we decide when a certain set of features is interesting enough to accommodate in a modeling language? While it is certainly important to ensure that a modeling language does not have any conceptual deficiencies for its users [27], it is possible to go overboard in this and end up with a language that is too large and complex too properly use. Clearly it would not do to incorporate explicit notational elements for every possible different conceptualization, as it would unnecessarily increase the cognitive complexity of the language. A good approach might be based in empirical findings, for example by looking at what (combinations of) often reoccurring features elicited among modelers are not accommodated, and analyze why that is the case.

While the scope of this paper is not to present an overview of such deficiencies based on our dataset (nor would the space constraints allow for such a treatment), we can discuss some of them as an example. For some concepts the primary features that have been discussed are all explicitly supported, like for instance goals being differentiated between those for which we know when we achieve them and those for which we don't. However, not every language allows for such distinctions, as ArchiMate 2.0 for example still does not incorporate the hard/soft goal distinction, even though it was based on a language (AMORE) which itself did. When it comes to events, the important features of intentionality are clearly supported in the most common languages dealing with events, like for instance BPMN's extensive dealing of triggers, and the possibility of modeling an unintentional event by using a 'None' type trigger. However, some of the features we found for resources are far less often supported by languages, like for example the identification of their nature in being material or immaterial. This does not seem to be explicitly supported in most modeling languages, usually being implicitly assumed to be a specific kind. A rare exception to this is ITML which at least enforced the distinction by using a hardware/software dichotomy where it is immediately clear whether a resource is a physical object or not. While not every feature listed once by an individual modeler should be part of each modeling language, it does seem to suggest that a combined effort of updating languages with the features important for their users would be a useful venture.

\section{B. Considerations}

The primary concern we have about the internal validity of our work and the presented results is the multi-lingual component of the answers, and the related difficulty in ensuring that meaning was correctly preserved during translation to a common language for the analysis phase. However, given that most responses were given by people fluent in both English and their native tongue, and the validation of the translations by multiple readers, we are confident that no errors were introduced in this phase.

Our external validity is ensured partially by the varied background of the participants: we have responses from people from multiple kinds of sectors (government, for-profits, educational institutes), and from different countries (USA, Netherlands, Germany, France, etc.). We aim to increase the external validity (that is, the generalizability of the sets of often repeated features) by incorporating another quantitative study that will further clarify the exact typicality of each of these features. a numerical typicality. However, because of the nature of the feature sets and the significant amount of terms that need to be investigated, this is a significant research effort which will take a longer running time in order to gain enough results.

\section{CONCLUSION}

We have shown the results of a study into what features conceptual modelers typically use to identify common modeling concepts. We discussed some interesting details of 
the data, including common modalities found in them, and the need for modeling languages to explicitly accommodate them. These features can be used as a guideline for what aspects are important to focus on during modeling sessions, as they represent the subtly different ways in which conceptual modelers interpret common modeling concepts, and might thus, unbeknownst to them, disagree with each other. In future work we will continue a study quantifying the exact typicality of the elicited features to create a graded structure, which, due to the significant size of the elicited datasets is a long-term research effort.

Acknowledgements.: This work has been partially sponsored by the Fonds National de la Recherche Luxembourg (www. fnr.lu), via the PEARL programme. Marc van zee is supported by the National Research Fund, Luxembourg.

\section{REFERENCES}

[1] M. M. Lankhorst, "Enterprise architecture modelling-the issue of integration," Advanced Engineering Informatics, vol. 18, no. 4, pp. 205 216, 2004.

[2] A. L. Opdahl and G. Berio, "Interoperable language and model management using the UEML approach," in Proceedings of the 2006 International Workshop on Global Integrated Model Management, New York, NY, USA, 2006, pp. 35-42.

[3] R. van Buuren, J. Gordijn, and W. Janssen, "Business case modelling for e-services," in 18 th Bled eConference eIntegration in Action, 2005.

[4] A. Persson and J. Stirna, "Why enterprise modelling? an explorative study into current practice," in Advanced Information Systems Engineering, ser. LNCS, Dittrich et al., Ed. Springer Berlin, 2001, vol 2068, pp. 465-468.

[5] D. Moody, "Theoretical and practical issues in evaluating the quality of conceptual models: current state and future directions," Data \& Knowledge Engineering, vol. 55, no. 3, pp. 243-276, 2005.

[6] C. M. Jonker, M. B. Riemsdijk, and B. Vermeulen, "Shared mental models," in Coordination, Organizations, Institutions, and Norms in Agent Systems VI, ser. Lecture Notes in Computer Science, M. Vos, N. Fornara, J. V. Pitt, and G. Vouros, Eds. Springer Berlin Heidelberg, 2011, vol. 6541, pp. 132-151.

[7] B. C. Malt, "Features and beliefs in the mental representation of categories," Journal of Memory and Language, vol. 29, no. 3, pp. 289 $-315,1990$

[8] P. Bloom, "Theory of artifact categorization," Cognition, vol. 66, no. 1, pp. $87-93,1998$.

[9] B. C. Malt and E. C. Johnson, "Do artifact concepts have cores?" Journal of Memory and Language, vol. 31, no. 2, pp. 195 - 217, 1992

[10] S. Harnad, "To cognize is to categorize: Cognition is categorization," in Handbook of Categorization, C. Lefebvre and H. Cohen, Eds. Elsevier, December 2005. [Online]. Available: http://eprints.soton.ac.uk/261725/

[11] P. Fettke, C. Houy, A.-L. Vella, and P. Loos, "Towards the reconstruction and evaluation of conceptual model quality discourses - methodical framework and application in the context of model understandability," in Enterprise, Business-Process and Information Systems Modeling, ser LNBIP, Bider et al., Ed. Springer Berlin Heidelberg, 2012, vol. 113, pp. 406-421.

[12] C. Houy, P. Fettke, and P. Loos, "Understanding understandability of conceptual models - what are we actually talking about?" in Conceptual Modeling, ser. LNCS, Atzeni et al., Ed. Springer, 2012, vol. 7532, pp. 64-77.

[13] S. Robinson, "Conceptual modeling for simulation: issues and research requirements," in Proceedings of the 38th conference on Winter simula tion, ser. WSC '06. Winter Simulation Conference, 2006, pp. 792-800.

[14] G. Stahl, "A model of collaborative knowledge-building," in Proc. of 4th Int. Conference of the Learning Sciences (ICLS 2000), Ann Arbor, MI, 2000, pp. 70-77.

[15] J. Sowa, "The Role of Logic and Ontology in Language and Reasoning," in Theory and Applications of Ontology: Philosophical Perspectives. Springer, 2010, pp. 231-263.
[16] J. Stirna, A. Persson, and K. Sandkuhl, "Participative enterprise modeling: Experiences and recommendations," in Advanced Information Systems Engineering, ser. LNCS, Krogstie et al., Ed. Springer Berlin Heidelberg, 2007, vol. 4495, pp. 546-560.

[17] C. Goddard, Words and the Mind. Oxford University Press, 2010, ch. Universals and Variation in the Lexicon of Mental State Concepts, pp 72-92.

[18] B. Wyssusek, "On ontological foundations of conceptual modelling," Scandinavian Journal of Information Systems, vol. 18, pp. 63-80, 2006.

[19] Y. Wand and R. Weber, "Research commentary: Information systems and conceptual modeling - a research agenda," Information Systems Research, vol. 13, no. 4, pp. 363-376, 2002.

[20] R. Barr and L. Caplan, "Category representations and their implications for category structure," Memory \& Cognition, vol. 15, no. 5, pp. 397418, 1987.

[21] D. J. T. van der Linden, S. J. B. A. Hoppenbrouwers, A. Lartseva, and H. A. Proper, "Towards an investigation of the conceptual landscape of enterprise architecture," in Enterprise, Business-Process and Information Systems Modeling, ser. LNCS, T. Halpin et al., Ed. Springer Berlin Heidelberg, 2011, vol. 81, pp. 526-535.

[22] J. C. Recker and A. Dreiling, "Does it matter which process modelling language we teach or use? An experimental study on understanding process modelling languages without formal education," in 18th Australasian Conference on Information Systems, Toowoomba, Australia, 2007, pp. 356-366.

[23] N. Guarino, "Formal ontology, conceptual analysis and knowledge representation," International Journal of Human-Computer Studies, vol. 43, pp. $625-640,1995$.

[24] I. Davies, P. Green, M. Rosemann, M. Indulska, and S. Gallo, "How do practitioners use conceptual modeling in practice?" Data \& Knowledge Engineering, vol. 58, no. 3, pp. 358 - 380, 2006.

[25] D. van der Linden and S. Hoppenbrouwers, "Challenges of identifying communities with shared semantics in enterprise modeling," in The Practice of Enterprise Modeling, ser. LNBIP, Sandkuhl et al., Ed. Springer Berlin Heidelberg, 2012, vol. 134, pp. 160-171.

[26] D. van der Linden and H. A. Proper, "Do conceptual modeling languages accommodate enough explicit conceptual distinctions?" in Short Paper Proceedings of the 6th IFIP WG 8.1 Working Conference on the Practice of Enterprise Modeling (PoEM 2013), Grabis et al., Ed., vol. 123, 2013, pp. 126-135.

[27] J. Recker, M. Rosemann, P. F. Green, and M. Indulska, "Do ontological deficiencies in modeling grammars matter?" MIS Quarterly, vol. 35, no. 1, pp. 57-79, 2011.

\section{APPENDIX}

The following appendices give the cleaned up sets of elicited features (with redundancies removed). They include each unique feature that was at least one practitioner gave as being in the top 5 of most important (i.e., salient) features for that particular concept.

\section{ACTOR}

has a name, has permissions, has a role, is a role, has skills, has capabilities, can perform an action, performs an action, stops an action, relaunches an action, schedules an action, cancels an action, belongs to a group, participates in a process, has a responsibility, can execute a task, can invoke a service, is assigned to a role, performs a task, performs an activity, has an assigned goal, uses resources, is responsible for tasks, initiates actions, is a responsible agent, can execute actions, can monitor the execution of actions, is capable of proactive action, is socially capable, is capable of assuming different roles, can perform actions, can receive information, can sense, is proactive, is deliberative, is an automatic thing, has responsibilities, can perform tasks, has access to a resource, can control a resource, can contribute to the fulfillment of goals, is composed of parts, performs actions, is human, is technical, can react to events, has to work under constraints, has rights, has access to resources, can fulfill multiple roles, carries knowledge, carries culture, is an active entity, is physical, displays behavior, can be human, can be a machine, carries out work, can carry responsibility, can receive responsibility, has an identity, has wishes, has desires, has competence, can act autonomously, executes work, requests work, is independent, uses services, uses active structures, is a person, is an organization, is responsible for a business function, is responsible for a business process, can trigger actions, administrates vision and principles, realizes vision and principles, realizes a goal, carries out actions, is non-human, is responsible, is accountable, is autonomous, is identifiable, is a computer, is a machine, is part of a business function, receives triggers, 
receives service requests, delivers a desired or agreed upon service, participates in a collaboration, is a company, is a person with a role in a company, has a concern, is a stakeholder, has a contractual relation, has relation to other actors, is a thing, does things, fulfills a role, has properties, acts in a context, is someone active in a context, is responsible in a context, has influence on a system, is reactive, can act, ensures the achievement of a result, is active, reacts to actions, makes decisions, always has an input, always has an output, has a quality level, has a role of a person, has a role of a machine acts, can be a department, can be an organization, can be a user, can be a system, can be a consumer of services, can offer services, has tasks, is absolute, has an intention, has an objective, has a goal, relates to a use case, relates to a scenario, relates to a function

\section{EVENT}

has a trigger, can be caught by something, has a related objective, is related to an actor, has a description, started on some time, started by some thing, has a precondition, has a postcondition, has a duration, has a cost, is recursive, has a cause, has consequences has a beginning, has an end, is triggered by something, can be temporal, has a name, can trigger an action, defines a transition from some state to some state, occurs in a process, has to be monitored, triggers a task, triggers a process, is atomic, is finite, is short, can cause a change, can be recognized by an actor, is unique, is identifiable, is instantaneous, is an occurrence, has some phenomena, is composed, is a change of state, occurs during an interval in time, is limited in time, can be planned, can be spontaneous, happens, triggers a business process, triggers a function, triggers a transformation, involves a least one subject, is executed by at least one subject, can be observed, results from a change, does not have a duration, has conditions, impacts other things, has a source, is a process end, is measurable, has relevance to business, triggers something, is the change of the state of an object, is related to a process, is related to a result, can trigger actions, is a fact, does not happen, is repeatable, can trigger a reaction, is predictable, occurs in the real world, can be anticipated upon, is a trigger, results in a case, is generated by a source, may be notified to listeners, is reported by an actor, report of an event is received by an actor, has a type, has a frequency, occurs at a specific time, triggers an action, has properties, influences a process flow, can disrupt a process, is caused by something, causes something, is unpredictable, can trigger a process, can occur spontaneously, has a timespan, triggers change in a situation, is spontaneous, can be caused by an action, is immutable, has an external cause, has an internal cause, occurs in the business layer, can trigger an activity, can be triggered, has a date, has a time

\section{GOAL}

has a time, has a result, has a related objective, is related to an actor, has a description, has a precondition, has a postcondition, has a cost, is defined by someone, has a name, has a type, has a relationship with a subgoal, has a relationship with an actor, is based on a need, is based on an objective, has to be reached during a process, is subjective, is relevant to someone, may be quantifiable, is agreed upon, is formulated according to the S.M.A.R.T. standard, is a desired state, is not currently the case, is an intention of an actor, has properties, has to be fulfilled, can be refined into a subgoal, can conflict with a goal, can be satisfied, has a property, is desired by an actor, is an objective is clear, has a limited scope, can be reached, is challenging, sensibility of which is attested by human ACTORS, can be supported by a person, is temporally valid, is a guideline, can be described quantitative, can be described qualitative, is formulated to be achieved in the middle-long-term, is delimited, has a related stakeholder, describes a desired outcome implies change, is the S.M.A.R.T. formulation of an aim, has a related actor, can be achieved, has a condition, has a purpose, gives direction, is thoughtful, is measurable, is owned by a stakeholder, should address a driver, becomes an evaluation criteria for future accomplishments, can be abstract, can be concrete, is future oriented, describes a desired state, describes a desired change, has value for an actor, can be quantitative, can be qualitative, has a source, is a desired future state, supports an organization, is formulated to support the strategy, fits within a broader framework, represents the directions given by something, is realizable within some time, is desired, is a future state, can be formulated according to the S.M.A.R.T. standard, has a timespan, has a KPI, is on some governance level, is tangible, is the desired result of an action, has to be achieved, is a reason for the existence of something, is a desired result, gives direction to an actor, describes a situation, is in the future, can be achieved in multiple ways, is planned, requires action to be achieved, makes strategy concrete, requires controlled actions to be achieved, is a state, has implications, can have subgoals, can have a strategy, can be broken down into parts

\section{PROCESS}

can be an instance of a method, is composite, groups tasks, has an endpoint, is executed atomically, is created by a modeler, has an input, has an output, is define through its components, is executable, is pauseable, is stoppable, is rewindable, is formally defined, is written in a modeling language, is bounded, is executed for a specific purpose, is a succession of actions, can be a chemical reaction, can be a corporate process, has steps, has a timeline, has related actors, has input, has output, is a set of related actions, reaches a goal, may involve roles, uses resources, has operating conditions, has a trigger event, may trigger another process, may depend on another process, can be owned, can be measured, has a trigger, is intangible, is composed of some tasks, has a sequence, has concurrency, is abstract, is detailed, has a start, has an end, has steps which are connected, can be conscious, can be passive, is specific to a problem, is specific to a solution, is not generic, is specific

\section{RESOURCE}

has features, has characteristics, can be available, needs permission for use, is used by an actor, has a related objective, has a description, has an owner, has a value, is composed, may be decomposed, is consumable, has an expiration date, satisfies a precondition, is material, is immaterial, is a person, is finite, is countable, has a name, has a label, has a type, can be human, can be material, is used for something, carries data, carries technology, carries knowledge, is used for actions, is used for behavior, has utility, has operational utility, has financial utility, has a benefit to something, can be abstract information, can be a representation of information, can be decomposed, can be accessed, can be controlled, can be software, can be hardware, is active, is passive, may be the realization of an EA artifact, classifies a resource, is scalable, is scarce, is exchangeable, is used by something, is valuable, has to be protected, has a capacity, has costs, is in a specific form, has a source, has a target audience, has a creation date, has a modification date, has a version, access is controlled, has a volume, is core, is non-core, is skilled, has structure, has boundaries, requires management, can be scarce, is a means to execute actions, is a means to initiate behavior, is transformed into a product, is consumed in a process, has a life cycle, is useful for some goal, has a price, is of a specific kind, exists in itself, has an availability, is human, is money, is input, is needed to realize output, is a means to perform an activity, can be a machine, is a quantity of human capacity, is a machine, is some amount of machine capacity, is needed for an activity, is optional, is required, has properties, is needed to achieve a goal, has availability, is difficult to manage, is a means of support for an actor, can have alternatives, is required for an activity, costs money, has a quality level, uses something, is an employee, is used up, has to be refilled, can be quantified, can be measured

\section{RESTRICTION}

is defined, is verifiable, has a type, has an owner, restricts something, limits something, is valid from some time, is valid until some time, is invalidated by someone, is cancelled by someone, has conditions, may have consequences, is defined by rules, has a name, is part of a policy, limits an actor's privileges, prevents an action, sets a precondition for an action, sets a postcondition for an action, sets a condition for an action, limits the permissible value range for an object, can be expressed numerically, is a regulation, is a limitation, is natural, is artificial, limits possible solutions, originates outside of its applicable area, is owned, needs to be discussed, has to be made explicit, should be formulated according to S.M.A.R.T., is a contribution of an actor to a result, is common, is measurable, is quantitative, can prevent an action, can limit possible solutions, has a source, is not allowed, is forbidden, has a source which is authoritative, has an operation domain, needs to be met during some period of time, can be temporal, can be determined by something, restricts the way something can be done, is a consequence of a higher principle, limits the behavior of an actor, has a motivation, violation may result in some punishment, has a level of generality, something might be compliant to a restriction, limits choices, may be represented as business rules, is a forbidden choice, is stipulated by others, is stipulated by the environment, limits a resource, can be absolute, limits an activity, is temporal, is situational, can influence a goal, can influence an event, is dogmatic behavior, is concrete, limits choice of scenarios, limits time, limits budget, limits available knowledge, is specific, is applicable to something, supports the realization of a goal, can have a quantitative value

\section{RESULT}

has a state, has data, has a related objective, has a related event, has a postcondition, has a precondition, satisfies a precondition, is measurable, has a name, is the output of a service, is the outcome of an action, meets a goal, is produced by an actor, is observable, can have an effect on something, is the consequence of an event, is output, is an outcome, can be aimed for, follows an action, is achieved by specific actions, can be described quantitatively, can be described qualitatively, is temporal, has value for someone, is delimited, is complete, has a quality, has related process, has a description, has a related actor, has a purpose, is the result of behavior, can affect a structure, is valued by a stakeholder, can be evidence of success, can be quantitative, can be qualitative, is achieved, is not achieved, exists after its achieving, is clearly described, is material, is immaterial, is a planned outcome of a process, can be part of a KPI, achievement can be monitored, is realized, is desired, achieving it can be a goal, has a type, is based on input values, is a product, is a service, has a form, has content, has volume, has a frequency, is a KPI, results after a process step, results after a process, can be composed, required to result from something, results from an action, enables other actions, results from an actor, is clear, can be input for an actor, contributes to a goal, has a property, has a benefit, has a value, results from a clear process, has a related value, has costs, is related to an activity, is a state of something, can be part of a process, associated with a process 